\title{
Effects of Music Therapy in Frail Elderlies: Controlled Crossover Study
}

\author{
Nobuyuki Murabayashi ${ }^{a}$ Takako Akahoshi $^{\mathrm{b}}$ Risa Ishimine ${ }^{\mathrm{c}}$ \\ Nobuko Saji $^{d}$ Chiyomi Takeda ${ }^{\text {e Hisako Nakayama }}{ }^{f}$ Megumi Noro ${ }^{g}$ \\ Hiroko Fujimoto $^{\text {h }}$ Megumi Misaki $^{i}$ Keiko Miyamotoj ${ }^{j}$ Yukiko Yamadak $^{k}$ \\ Itaru Kohyal Mayu Kondo ${ }^{m}$ Haruyasu Yamaguchin ${ }^{n}$ Daisuke Sasaki ${ }^{\circ}$ \\ Yasuji Muraip \\ aPsychosomatic Arts Clinic Osaki, Tokyo, Japan; ${ }^{b}$ Tokyo Music Volunteer Society, Tokyo, \\ Japan; ' Music Therapy ARIA, Tachikawa, Japan; ' Miyagi University, Sendai, Japan; ' Kamase

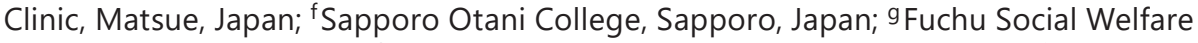

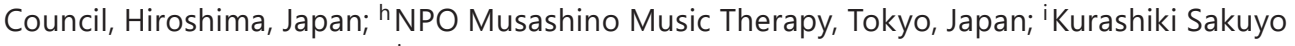 \\ University, Kurashiki, Japan; 'Kasanomisaki Music Therapy Research, Ishikawa, Japan; \\ kNishinomiya Music Therapy Association, Nishinomiya, Japan; 'Kanagawa Rehabilitation \\ Hospital, Atugi, Japan; mTokai University, Isehara, Japan; ${ }^{\mathrm{n}}$ Gunma University, Maebashi, \\ Japan; ${ }^{\circ}$ Geriatric Health Services Facility La foret Tendo, Tendo, Japan; ${ }^{P}$ Seitoku University, \\ Matudo, Japan
}

\section{Keywords}

Controlled crossover trial · Frail elderly individuals · Music therapy · Physical and emotional behaviors

\begin{abstract}
Background: Music therapy (MT) may exert beneficial preventive effects in frail elderly individuals in psychophysiological health. Objectives: One hundred and fifteen frail elderly individuals (65-89 years old) participated. Methods: Subjects were randomly divided into 2 groups for a controlled crossover trial. The MT-first group participated in group sessions of 45-50 min conducted by a certified music therapist for 12 weeks, followed by a 12-week waiting period. The waiting-first group participated in the reverse order. Cognitive function was assessed by the Verbal Fluency Test (VFT) and Yamaguchi Kanji Symbol Substitution Test (YKSST). Physical function was assessed by the Timed Up and Go test (TUG). Psychophysical health was assessed by the Geriatric Depression Scale 15-item version (GDS-15), General Health Questionnaire 12-item version (GHQ-12), Instrumental Activity of Daily Living (IADL) scale, and Life Space Assessment (LSA) scale. We also evaluated the outcome of MT using the Clinical Global Impressions of Improvement (CGI-I) scale. Results: After 12 weeks of MT, sig-
\end{abstract}


nificant improvements were observed in TUG, GDS-15, and GHQ-12 scores. However, no improvements were noted in other scores. CGI-I scores revealed the significant benefits of MT. Conclusions: MT may improve physical function, depressive mood, and quality of life in frail elderly individuals.

(c) 2019 The Author(s)

Published by S. Karger AG, Basel

\section{Introduction}

Japan is one of the fastest aging societies in the world, with the number of elderly individuals who need nursing care rapidly increasing [1]. In 2017, this number was estimated to be over 6 million. It is important to maintain independence in daily life activities and prevent the progression of functional decline from an early stage in frail elderly individuals. Although there is no clear consensual definition of a frail condition, it is a clinical condition that is associated with a high risk of functional decline [2]. Frailty is highly prevalent in the elderly and increases their vulnerability to adverse health outcomes such as disabilities and the need for long-term care. In Japan, the objective of long-term care service programs (Kaigo-yobo) is "the reduction or prevention of deterioration of conditions for which a person requires continuous care for the whole or a part of basic movements during daily activities, or for a person who has difficulty performing daily activities due to physical or mental disabilities" [3], as stated in the Long-Term Care Insurance Act. The Ministry of Health, Labour and Welfare (MHLW) has implemented a care policy for the elderly that addresses six areas of need: the improvement of physical activities, nourishment, and oral cavity hygiene; support for the elderly living alone; and the prevention of dementia and depression.

In a systematic literature review article, Stuck et al. [4] suggested that the strongest predictive indications for increased risk in functional decline were cognitive impairment, depression, disease burden, increased or decreased body mass index, lower extremity functional limitations, low frequency of social contact, low level of physical activity, and no alcohol use compared to moderate use. Preventive factors for dementia in the elderly include lifestyle-related factors such as eating habits, physical activity, no smoking, and leisure activity [5]. Verghese et al. [6] reported that among leisure activities, reading, playing board games, playing musical instruments, and dancing reduced the risk of dementia over a 5-year follow-up period. Our previous pilot open study of elderly living at long-term care facilities suggests that music therapy (MT) improved cognitive function, activities associated with daily life, and quality of life (QOL), thereby contributing to improvements in mental and physical health [7].

Previously, the effectiveness of MT has been investigated in healthy elderly individuals. Cohen et al. [8] investigated the impact of choir singing on physical and mental health; the intervention group had fewer doctor visits, less medication use, fewer falls, better morale, and less loneliness. Choir singing was also found to have a positive influence on both emotions and immune competence [9].

MT has been reported to improve cognitive function and moods [10]. Listening to music or musical activity with instruments or dancing has been shown to have cognitive benefits $[11,12]$. Takahashi et al. [13] also reported that MT maintained cognitive function and QOL.

In a randomized controlled trial (RCT) on mild cognitive impairment, active MT reduced the progression rate of dementia over 2 years [14]. Furthermore, physical exercise combined with music exerted more positive effects on visuospatial and nonverbal cognitive function in the elderly than exercise alone [15]. This study performed physical exercise interventions with and without music in 119 community-dwelling normal elderly individuals for 1 year, although it is unclear how many of the participants were frail. 
Särkämö et al. [16] conducted an RCT on participants with mild-moderate dementia. These participants were randomly assigned to a singing group (SG), music listening group (MLG), and usual care control group. SG and MLG engaged in a group-based music program (1.5 h/session) once a week for 10 weeks. SG and MLG improved mood, orientation, remote episodic memory, attention, executive function, and general condition. SG also improved short-term and working memory and caregiver well-being, while MLG had a positive effect on QOL.

Although a number of studies have investigated the effects of MT on elderly individuals with dementia, few have examined frail elderly individuals $[15,17]$. Furthermore, the outcome measures of these studies were cognitive function, mood, and QOL, with few studies referring to motor functions [17].

Due to the lack of evidence from rigorous data-based studies, MT is not yet accepted as one of the approved preventive methods for long-term care. Therefore, the aim of the present controlled crossover study was to investigate whether MT improves cognitive and physical functions, mood states, and QOL. Our current results will support MT as an effective method for preventive care in frail elderly individuals.

\section{Materials and Methods}

\section{Participants and Randomization}

We informed 122 participants from 11 therapy rooms about the project between August 2013 and September 2014. The sample size was calculated using the findings of a previous study [7]. Using a significance level of 0.05 with a power of 0.80 and 2-sided hypothesis testing, a sample size of 51 was considered to be necessary in each group. Accordingly, predicting a drop-out rate of approximately $20 \%, 122$ people were invited to participate in the study.

The inclusion criteria were an age range of 65-89 years, having one or more care needs regarding social withdrawal, dementia, or depression as assessed using a frailty checklist (Kihon Checklist) based on the Japanese long-term care insurance system, which consists of 25 items (Appendix), and/or the Category of "Needed Support Condition" support level 1 or 2 as assessed by an ordinance of the MHLW [1,3]. These participants were in the pre-stage of care-need support according to the basic checklist and assessment sheet for long-term care prevention administered by municipal governments [18]. This checklist has acceptable reliability and validity [19].

Each participant was randomly assigned to an MT-first group or waiting-first group for 12 weeks (the first period) and was then assigned to the alternate group (the second period). A 4-week washout period was established between the crossover to reduce potential carryover effects, which was based on studies that demonstrated the effects of a music intervention dissipating between 1 and 4 weeks after an intervention [20]. Participants in the waiting-first group were informed that they would receive MT intervention after the waiting period.

The randomized allocation of participants to either group was conducted by the random choice of code-enclosed envelopes. None of the staff or participants had access to the randomization code during the study.

\section{Music Therapy}

This study took place in 11 therapy rooms, and two qualified music therapists performed the MT sessions. Each MT group consisted of $5.6 \pm 1.6$ participants, and sessions were conducted for 45-50 min once a week for 12 weeks. The participants then crossed over into 
the opposite arm and the waiting protocol was conducted for another 12 weeks. A participant's attendance register was taken at the start of each session.

MT sessions basically consisted of four parts: (1) introduction (musical greeting and physical exercise with music); (2) exposition (singing seasonable songs); (3) development (singing familiar songs, instrumental activities, and rehabilitative activities); and (4) a closing part (reminiscence and listening). All sessions were conducted according to this program, while the selection of music, musical activities, and rehabilitative activities was left to each therapist's decision.

The therapist initially played music or a rhythmic pattern to the participants and led them to join in. When participants sang and clapped their hands spontaneously at their own pitch and tempo, the therapists adjusted the pitch and tempo of the keyboard to match their playing and singing and accompanied their expressions. Small instruments of resonating sound, such as tone chimes and music bells, were selected for instrumental activities to allow the participants to listen to their sounds connecting with others and to feel part of the group.

\section{Outcome Measures}

At the first baseline and 12 (first post), 16 (2nd baseline), and 28 (2nd post) weeks after, three functions were assessed in participants using 8 outcome measures. Cognitive function was measured by the Verbal Fluency Test (VFT) and Yamaguchi Kanji Symbol Substitution Test (YKSST), physical function by the Timed Up and Go test (TUG), and psychophysical health by the Geriatric Depression Scale 15-item version (GDS-15), General Health Questionnaire 12-item version (GHQ-12), Instrumental Activity of Daily Living (IADL), and Life Space Assessment (LSA).

At the VFT, subjects recalled the names of as many animals as possible for $1 \mathrm{~min}$. The YKSST is similar to the digit-symbol substitution test of the Wechsler Adult Intelligence ScaleIII (WAIS-III). The mean \pm SD YKSST score in healthy elderly subjects was $46.9 \pm 10.9$ ( $n=$ 170, age between 60 and 80 years) [21]. The TUG measured the time needed from standing up from a chair, walking $3 \mathrm{~m}$, turning around, walking back to the chair, and sitting down. The GDS-15, GHQ-12, IADL, and LSA were Japanese versions, and they have acceptable reliability and validity [22-24].

We also evaluated the overall outcome of MT using the Clinical Global Impressions of Improvement (CGI-I), and incorporated assessments by the music therapists by calculating the number of participants who had a "very much improved "or "much improved" score. Outcome measures were assessed by the same staff at each visit, whenever possible. Assessors were blinded when they assessed the outcome measures. Each music therapist was trained on how to conduct evaluations with videotapes. Adverse events were monitored and reported throughout the study. A flow diagram of the recruitment and study procedures is shown in Figure 1.

\section{Data Analysis}

All data were analyzed with per protocol crossover analysis method using StatsDirect Ver. 3.0 (StatsDirect Ltd.). In the analysis, each outcome measure was calculated as a change in the score from the baseline. A two-sided $p$ value of $<0.05$ was considered to be significant. The absence of a significant $(p>0.1$ ) period-effect and treatment-period interaction permits the use of significance to assess effects in the MT-first group with those in the waiting-first group. 


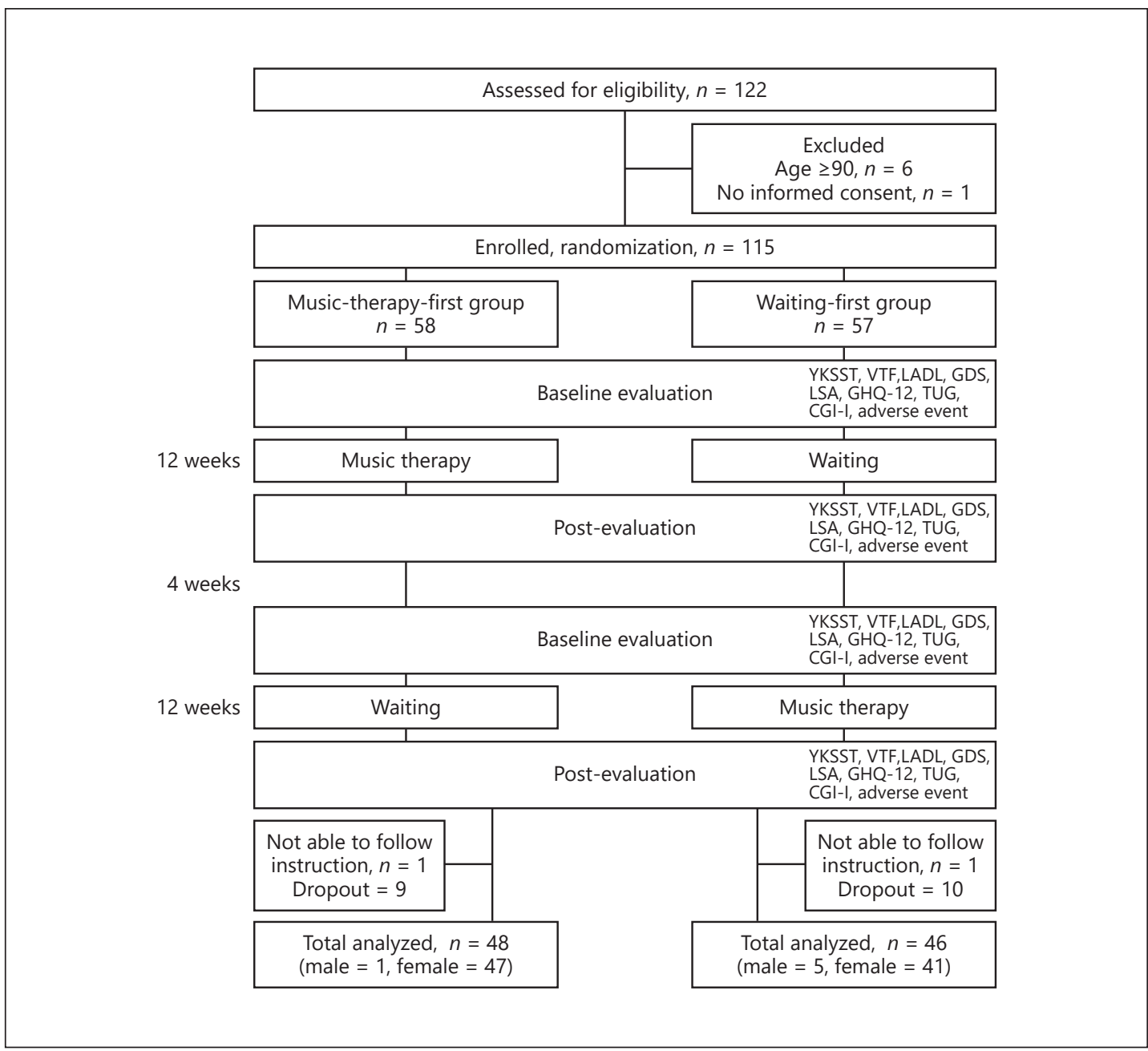

Fig. 1. Participant flow in a consort chart. Data are expressed as a flow diagram of the study. A total of 122 participants were recruited; 115 participants who met the criteria of the study were randomly divided into the music-therapy-first group or waiting-first group. During the 28 weeks of the trial, 94 participants completed the program.

\section{Results}

\section{Participant Characteristics}

Participant characteristics are shown in Table 1. We initially recruited 122 participants; 6 did not match the inclusion criteria and were excluded, while 1 withdrew consent to participate. Thus, we obtained a data set from 115 participants. Then, the data sets from 21 participants were excluded from either phase one or phase two of the study due to an attendance rate of less than $60 \%$ or physical issues such as pneumonia in either arm one or two of the study. Consequently, 48 participants were assigned to the MT-first group and 46 participants to the waiting-first group. Most participants were female (93.6\%). The MT-first group and waiting-first group had similar baseline parameters including age, gender, and mean careneed stage (Table 2). There were smaller samples compared with the necessary sample size (3 participants in the MT-first group and 5 in the waiting-first group), although these did not appear to affect statistical comparisons. 
Table 1. Characteristics of participants $(n=94)$

\begin{tabular}{lcc}
\hline & $\begin{array}{l}\text { Music-therapy-first group } \\
(n=48)\end{array}$ & $\begin{array}{l}\text { Waiting-first group } \\
(n=46)\end{array}$ \\
\hline Male:female, $n$ & $1: 47$ & $5: 41$ \\
Care-need stage & $1.6 \pm 0.9$ & $1.7 \pm 0.9$ \\
Experience of music therapy, $n$ & 12 & 16 \\
Average age, years & $80.9 \pm 5.6$ & $81.7 \pm 5.5$ \\
\hline
\end{tabular}

There were no significant differences between the 2 groups.

\section{Intervention Effects}

No significant differences were observed either in the period-effect or treatmentperiod interaction. Significant improvements were noted between the MT-first group and waiting-first group for TUG (effect magnitude -0.64), GDS-15 (effect magnitude -0.75), and GHQ-12 (effect magnitude -0.95) scores (Table 2). In the crossover study, the CGI-I responder rate was significantly better in the MT-first group than in the waiting-first group $(p<0.05)$ (Table 3). Throughout the study, no serious adverse events were noted in either group.

\section{Discussion}

There is an increasing need for mental and physical exercise as preventive care to maintain overall health and function in the elderly. MT has been suggested to reduce social, emotional, and physiological disabilities in frail elderly individuals [25]. Although many studies have reported the beneficial effects of MT, few have been conducted on preventive care in frail elderly individuals, and MT has yet to be accepted as one of the preventive methods of long-term care service programs (Partial Revision of the LongTerm Care Insurance Act, etc., to strengthen Long-Term Care Service Infrastructure, MHLW). Vink et al. [26] collected and analyzed RCT on MT in elderly individuals with dementia and reported that the methodological quality of these studies was too poor to draw conclusions, particularly considering the small sample sizes and insufficient statistical analyses. The majority of previous studies used smaller sample sizes than that in the present study, in which 94 frail elderly individuals were recruited from 11 therapy rooms and treated using the same protocol. MT was provided once weekly, for a total of 12 weeks.

Ueda et al. [27] performed a meta-analysis on the effects of MT from 20 selected studies. The average time of each intervention session was $36 \mathrm{~min}$, conducted 2 or 3 times per week, for 10 weeks. In the present study, each intervention lasted for 45-50 min, and was conducted once a week over a 12-week period. After psychological and rehabilitative approaches to active MT, Raglio et al. [28] concluded that studies on the efficacy of active MT have a firm empirical foundation. The strength of our study was that MT was applied based on a psychological and rehabilitative protocol using a randomized crossover design and multiple assessment criteria, with a large number of participants. The multiple assessment tools applied were as follows: cognitive function was assessed by VFT and YKSST, physical function by TUG, and psychophysical health by GDS-15, GHQ-12, the IADL scale, and LSA scale. A key 
Table 2. Comparison of effects between the music-therapy-first group and waiting-first group on cognitive and physical function a Comparison of effects

\begin{tabular}{|c|c|c|c|c|c|c|c|}
\hline & \multicolumn{2}{|c|}{ Mean changes from baseline } & & \multirow[t]{2}{*}{ SE } & \multirow[t]{2}{*}{$t$} & \multirow[t]{2}{*}{ df } & \multirow[t]{2}{*}{$p$} \\
\hline & first period & second period & & & & & \\
\hline \multicolumn{8}{|l|}{$\begin{array}{l}\text { Cognitive function } \\
V T F\end{array}$} \\
\hline Music-therapy-first group & 0.58 & 0.63 & period effect & 0.92 & 0.02 & 93 & 0.98 \\
\hline Waiting-first group & 0.53 & 0.46 & $\begin{array}{l}\text { treatment-period interaction } \\
\text { relative effectiveness } \\
\text { treatment effect }\end{array}$ & $\begin{array}{l}0.94 \\
0.46 \\
0.92\end{array}$ & $\begin{array}{l}0.22 \\
0.11 \\
0.11\end{array}$ & $\begin{array}{l}93 \\
94 \\
93\end{array}$ & $\begin{array}{l}0.82 \\
0.91 \\
0.91\end{array}$ \\
\hline \multicolumn{8}{|l|}{ YKSST } \\
\hline Music-therapy-first group & 2.46 & 0.44 & period effect & 1.98 & 2.04 & 93 & 0.04 \\
\hline Waiting-first group & 1.30 & -0.72 & $\begin{array}{l}\text { treatment-period interaction } \\
\text { relative effectiveness } \\
\text { treatment effect }\end{array}$ & $\begin{array}{l}2.32 \\
1.00 \\
1.99\end{array}$ & $\begin{array}{l}1.13 \\
0.02 \\
0.00\end{array}$ & $\begin{array}{l}93 \\
94 \\
93\end{array}$ & $\begin{array}{l}0.25 \\
0.98 \\
1.00\end{array}$ \\
\hline \multicolumn{8}{|l|}{ Physical function } \\
\hline Music-therapy-first group & -0.85 & 0.17 & period effect & 0.58 & -1.3 & 93 & 0.20 \\
\hline Waiting-first group & 0.06 & 0.27 & $\begin{array}{l}\text { treatment-period interaction } \\
\text { relative effectiveness } \\
\text { treatment effect }\end{array}$ & $\begin{array}{l}0.64 \\
0.29 \\
0.58\end{array}$ & $\begin{array}{l}-1.69 \\
-2.21 \\
-2.20\end{array}$ & $\begin{array}{l}93 \\
94 \\
93\end{array}$ & $\begin{array}{l}0.09 \\
0.02 \\
0.03\end{array}$ \\
\hline \multicolumn{8}{|l|}{$\begin{array}{l}\text { Psychological health } \\
G D S-15\end{array}$} \\
\hline Music-therapy-first group & -0.56 & -0.42 & period effect & 0.73 & 1.67 & 93 & 0.10 \\
\hline Waiting-first group & 0.91 & -0.45 & $\begin{array}{l}\text { treatment-period interaction } \\
\text { relative effectiveness } \\
\text { treatment effect }\end{array}$ & $\begin{array}{l}0.63 \\
0.37 \\
0.73\end{array}$ & $\begin{array}{l}-2.28 \\
-2.04 \\
-2.08\end{array}$ & $\begin{array}{l}93 \\
94 \\
93\end{array}$ & $\begin{array}{l}0.02 \\
0.04 \\
0.04\end{array}$ \\
\hline \multicolumn{8}{|l|}{ GHQ-12 } \\
\hline Music-therapy-first group & -1.23 & 0.04 & period effect & 0.68 & -0.93 & 93 & 0.35 \\
\hline Waiting-first group & 0.40 & -0.23 & $\begin{array}{l}\text { treatment-period interaction } \\
\text { relative effectiveness } \\
\text { treatment effect }\end{array}$ & $\begin{array}{l}0.71 \\
0.34 \\
0.68\end{array}$ & $\begin{array}{l}-1.92 \\
-2.83 \\
-2.82\end{array}$ & $\begin{array}{l}93 \\
94 \\
93\end{array}$ & $\begin{array}{l}0.06 \\
0.01 \\
0.01\end{array}$ \\
\hline \multicolumn{8}{|l|}{$\overline{I A D L}$} \\
\hline Music-therapy-first group & 0.13 & -0.10 & period effect & 0.39 & -0.68 & 93 & 0.50 \\
\hline Waiting-first group & 0.40 & -0.23 & $\begin{array}{l}\text { treatment-period interaction } \\
\text { relative effectiveness } \\
\text { treatment effect }\end{array}$ & $\begin{array}{l}0.44 \\
0.20 \\
0.39\end{array}$ & $\begin{array}{r}-1.79 \\
1.34 \\
1.33\end{array}$ & $\begin{array}{l}93 \\
94 \\
93\end{array}$ & $\begin{array}{l}0.08 \\
0.18 \\
0.19\end{array}$ \\
\hline \multicolumn{8}{|l|}{$\overline{L S A}$} \\
\hline Music-therapy-first group & -2.58 & 0.55 & period effect & 5.98 & -1.26 & 93 & 0.21 \\
\hline Waiting-first group & -3.72 & 0.69 & $\begin{array}{l}\text { treatment-period interaction } \\
\text { relative effectiveness } \\
\text { treatment effect }\end{array}$ & $\begin{array}{l}6.46 \\
3.00 \\
5.98\end{array}$ & $\begin{array}{r}0.15 \\
-0.20 \\
-0.21\end{array}$ & $\begin{array}{l}93 \\
94 \\
93\end{array}$ & $\begin{array}{l}0.88 \\
0.84 \\
0.83\end{array}$ \\
\hline
\end{tabular}

b Effect magnitude

\begin{tabular}{ll}
\hline VTF & $-0.06(-0.97 \sim 0.87)$ \\
YKSST & $-0.00(-1.97 \sim 1.97)$ \\
TUG & $-0.64(-1.22 \sim 0.06)$ \\
GDS-15 & $-0.75(-1.47 \sim 0.03)$ \\
GHQ-12 & $-0.95(-1.63 \sim-0.28)$ \\
IADL & $0.26(-0.13 \sim 0.65)$ \\
LSA & $0.64(-5.3 \sim 6.6)$
\end{tabular}

Data are expressed as effect of music therapy using crossover analysis. Data in parentheses are 95\% confidence intervals. Significant improvements were noted between the MT-first group and waiting-first group for TUG, GDS-15, and GHQ-12 scores. SE, standard error; VTF, Verbal Fluency Test; YKSST, Yamaguchi Kanji Symbol Substitution Test; TUG, Timed Up and Go Test; GDS-15, Geriatric Depression Scale-15; GHQ-12, General Health Questionnaire-12; IADL, Instrumental Activity of Daily Living; LSA, Life Space Assessment Test. 
Table 3. Clinical Global Impressions of Improvement (CGI-I) scale responder rate $(n=94)$

\begin{tabular}{llc}
\hline Study group & Participants, $n$ & Responders, $n(\%)$ \\
\hline Music-therapy-first group & 48 & $22(45.8)$ \\
Waiting-second group & 48 & $7(14.5)$ \\
Music-therapy-second group & 46 & $15(32.6)$ \\
Waiting-first group & 46 & $2(4.3)$ \\
Music therapy group & 94 & $37(39.7)$ \\
Waiting group & 94 & $9(9.6)$ \\
\hline & responder score: $<3$ & nonresponder score: $<3$ \\
\hline
\end{tabular}

The CGI-I responder rate was significantly better in the music-therapy-first group than in the waiting-first group.

result in the present study is the improvement of physical function in TUG, and psychophysical health in GDS and GHQ.

Only a few studies have investigated the efficacies of MT in elderly individuals who are assessed as being frail at the pre-stage of care-need support. Kamioka et al. [29] analyzed systematic reviews based on RCT of music interventions and provided evidence of the positive effects of MT for schizophrenia and/or serious mental disorders for global and social functioning, Parkinson's disease for gait and related activities, depressive symptoms, and sleep quality. They proposed that MT has a direct effect on improvements in mental health, reductions in pain, communication with other individuals, and QOL. Vasionyte and Madison identified 19 studies based on RCT or clinical control trials using meta-analyses and music interventions demonstrating prominent positive effects on behavioral, cognitive, and physiological outcome measures as well as medium effects on affective measures [30]. They concluded that music interventions appear to be effective and have the potential to improve the QOL of patients with dementia.

Zhang et al. [31] performed a meta-analysis on the effects of MT for dementia from 34 selected studies. They reported evidence that MT decreased disruptive behavior, anxiety, and depression, and improved cognitive function and QOL. Although the severity of cognitive deterioration differed among participants in these studies, positive effects on cognitive function were reported in the elderly $[15,16]$.

On the other hand, Ueda et al. [27] reported that the effects of MT on depression and behavior were unsubstantiated, lacking in empirical evidence to verify any claim for the beneficial effects of MT on cognitive function and ADL. van der Steen et al. [32] performed a metaanalysis on the effects of MT for dementia from 17 selected studies. They concluded that MT reduced depressive symptoms, but had little or no effect on emotional well-being, overall behavioral problems, and cognition, and the quality of evidence on anxiety and social behavior was very low. These differences in research outcomes underscore the importance of ongoing studies regarding the effectiveness of MT in frail elderly individuals.

We demonstrated that MT improved some physical and emotional parameters in frail elderly individuals. Since MT is not yet formally recognized in Japan, it is important to facilitate MT by promoting the use and spread of these effective nonpharmacological approaches, which are indeed applicable to preventive care for frail elderly individuals at low cost and without side effects.

The study has certain limitations. First, $94 \%$ of the participants in this study were women in their 80s. Further studies may clarify whether MT might show the same effects for subjects 
with a wider age range and male subjects. Second, MT had no effect on cognitive function in this study, which is inconsistent with previous findings [15, 30,31]. Third, we did not evaluate what kind of musical activity (singing, playing musical instruments, listening, composing, or exercising) was most effective among the participants. Further studies are needed to evaluate the effects of MT as preventive care for frail elderly individuals.

\section{Conclusion}

We demonstrated significant improvements in TUG, GDS, and GHQ following MT. These results suggest that MT performed by a credentialed music therapist improves some physical and emotional parameters in frail elderly individuals and may be regarded as a useful and safe preventive method for long-term care service programs.

\section{Acknowledgments}

This research was conducted by the Research Project of the Japanese Music Therapy Association. We received financial support from the Japanese Music Therapy Association. The funder had no role in study design, data collection and analysis, decision to publish, or preparation of the manuscript.

\section{Statement of Ethics}

This study was conducted in accordance with the revised Declaration of Helsinki. The study protocol was approved by the IRB of the Institutional Ethics Committees of the Japanese Music Therapy Association in February 2013 (IRB No.001-2013). Written informed consent was obtained from all participants before every procedure.

\section{Disclosure Statement}

The authors have no conflicts of interest to declare.

\section{Funding Sources}

This work was not supported by any external funding agency.

\section{Author Contributions}

N.M., I.K., M.K., H.Y., D.S., and Y.M. designed the study and wrote the protocol. T.A., R.I., N.S., C.T., H.N., M.N., H.F., M.M., K.M., and Y.Y. collected and processed the data. D.S. performed the statistical analysis. N.M. wrote the first draft of the manuscript. All authors contributed to and approved the final paper. 


\section{Appendix}

Kihon Checklist (Ministry of Health, Labour and Welfare)

Basic health check list for those over 65 years old (English)

\begin{tabular}{|l|}
\hline Date \\
\hline Name \\
\hline Year \& date of birth \\
\hline
\end{tabular}

\begin{tabular}{|c|c|c|c|c|}
\hline 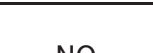 & & & Circle eit & one. \\
\hline \multirow[t]{5}{*}{1 daily life } & 1 & Do you normally travel by bus or train by yourself? & O Yes & 1 No \\
\hline & 2 & Do you go out and by daily necessities by yourself? & o Yes & 1 No \\
\hline & 3 & Do you manage your own deposits and savings at the bank? & o Yes & $1 \mathrm{No}$ \\
\hline & 4 & Do you often go out to visit your friends? & O Yes & 1 No \\
\hline & 5 & $\begin{array}{l}\text { Do you consult with your family or friends about their } \\
\text { problems? }\end{array}$ & O Yes & 1 No \\
\hline \multirow[t]{5}{*}{$\begin{array}{c}2 \text { physical } \\
\text { ability }\end{array}$} & 6 & Are you able to go upstairs without holding rail or wall? & O Yes & 1 No \\
\hline & 7 & Are you able to stand up from the chair without any aids? & o Yes & $1 \mathrm{No}$ \\
\hline & 8 & Are you able to keep walking for about 15 minutes? & o Yes & $1 \mathrm{No}$ \\
\hline & 9 & Have you fallen during the past year? & 1 Yes & 0 No \\
\hline & 10 & Do you worry about falling down? & 1 Yes & 0 No \\
\hline 3 nutrition & 11 & Have you lost more than $2 \sim 3 \mathrm{~kg}$ in the past 6 months? & 1 Yes & 0 No \\
\hline
\end{tabular}


Murabayashi et al.: Music Therapy for Frail Elderlies

\section{References}

1 Kuriyama S, Nakaya N, Ohmori-Matsuda K, Shimazu T, Kikuchi N, Kakizaki M, et al. The Ohsaki Cohort 2006 Study: design of study and profile of participants at baseline. J Epidemiol. 2010;20(3):253-8.

2 Xue QL, Bandeen-Roche K, Varadhan R, Zhou J, Fried LP. Initial manifestations of frailty criteria and the development of frailty phenotype in the Women's Health and Aging Study II. J Gerontol A Biol Sci Med Sci. 2008 Sep; 63(9):984-90.

3 Murayama H, Nishi M, Shimizu Y, Kim MJ, Yoshida H, Amano H, et al. The Hatoyama Cohort Study: design and profile of participants at baseline. J Epidemiol. 2012;22(6):551-8.

4 Stuck AE, Walthert JM, Nikolaus T, Büla CJ, Hohmann C, Beck JC. Risk factors for functional status decline in community-living elderly people: a systematic literature review. Soc Sci Med. 1999 Feb;48(4):445-69.

5 Plassman BL, Williams JW Jr, Burke JR, Holsinger T, Benjamin S. Systematic review: factors associated with risk for and possible prevention of cognitive decline in later life. Ann Intern Med. 2010 Aug; 153(3):18293.

6 Verghese J, Lipton RB, Katz MJ, Hall CB, Derby CA, Kuslansky G, et al. Leisure activities and the risk of dementia in the elderly. N Engl J Med. 2003 Jun;348(25):2508-16.

7 Murabayashi N, Saji N, Nakayama H, Hujimoto H, Miyamoto K, Kohya I, et al. Efficacy of music therapy on mental and physical health of elderly people in long-term care facilities: a pilot project. JMTA J. 2014;14: 58-65.

8 Cohen GD, Perlstein S, Chapline J, Kelly J, Firth KM, Simmens S. The impact of professionally conducted cultural programs on the physical health, mental health, and social functioning of older adults. Gerontologist. 2006 Dec;46(6):726-34.

9 Kreutz G, Bongard S, Rohrmann S, Hodapp V, Grebe D. Effects of choir singing or listening on secretory immunoglobulin A, cortisol, and emotional state. J Behav Med. 2004 Dec;27(6):623-35.

10 Sekiya M, Isoda K. Improvement effects of continuous, long-term active music therapy on cognitive function and emotions of at-home senior citizens. JMTA J. 2005;5:198-206.

11 Hanna-Pladdy B, MacKay A. The relation between instrumental musical activity and cognitive aging. Neuropsychology. 2011 May;25(3):378-86.

12 Kattenstroth JC, Kolankowska I, Kalisch T, Dinse HR. Superior sensory, motor, and cognitive performance in elderly individuals with multi-year dancing activities. Front Aging Neurosci. 2010 Jul;2:e1-9.

13 Takahashi T, Takano Y. Music therapy for dementia prevention focusing on handbell activities. JMTA J. 2010; 10:202-9.

14 Okumura Y, Okumura A, Matumoto J, Momma Y, Toyoshima Y, Shimoda J, et al. The cognitive music therapy for mild cognitive impairment. JMTA J. 2010;10:28-36.

15 Sato M, Ogawa J, Tokita T,Nakaguchi N, Nakao K, Kida H et al. The effects of physical exercise with music on cognitive function of elderly people: Mihama-Kiho project. PLoS One. 2014 Apr;9(4):e95230.

16 Särkämö T, Tervaniemi M, Laitinen S, Numminen A, Kurki M, Johnson JK, et al. Cognitive, emotional, and social benefits of regular musical activities in early dementia: randomized controlled study. Gerontologist. 2014 Aug;54(4):634-50.

17 Yokoi K, Kunitomo T, Shimada J, Tuzi R. An empirical study on effective anti-dementia project: comparative investigation of approaches with music therapy and recrational activities. J Hum Nurs Stud. 2007; 5: 81-8.

18 Ministry of Health, Labour and Welfare. [Long-term care prevention project manual]. 2012. pp. 1-13. Japanese.

19 Satake S, Senda K, Hong YJ, Miura H, Endo H, Sakurai T, et al. Validity of the Kihon Checklist for assessing frailty status. Geriatr Gerontol Int. 2016 Jun;16(6):709-15.

20 Cooke M, Moyle W, Shum D, Harrison S, Murfield J. A randomized controlled trial exploring the effect of music on quality of life and depression in older people with dementia. J Health Psychol. 2010 Jul;15(5): 765-76.

21 Yamaguchi T, Maki Y, Kaiho A. Yamaguchi kanji symbol substitution test as a scale of executive function in old people for preventing dementia in community. Jap J Geriatr Psychiatry. 2011;22:587-94.

22 Fukunishi I. The assessment of cut-off point of the General Health Questionnaire (GHQ) in the Japanese version. Shinri Rinshou. 1990;3:228-34.

23 Koyano W, Shibata H, Haga H, Suyama Y, Nakazato K. Measurement of competence in the elderly living at home: development of an index of competence. Jap J Public Health. 1987;34:109-114.

24 Harada K, Shimada H, Sawyer P, Asakawa Y, Nihei K, Kaneya S, et al. [Life-space of community-dwelling older adults using preventive health care services in Japan and the validity of composite scoring methods for assessment]. Nihon Koshu Eisei Zasshi. 2010 Jul;57(7):526-37. Japanese.

25 Kneafsey R. The therapeutic use of music in a care of the elderly setting: a literature review. J Clin Nurs. 1997 Sep;6(5):341-6.

26 Vink AC, Bruinsma MS, Scholten RJ. Music therapy for people with dementia. Cochrane Database Syst Rev. 2004;(3):CD003477.

27 Ueda T, Suzukamo Y, Sato M, Izumi S. Effects of music therapy on behavioral and psychological symptoms of dementia: a systematic review and meta-analysis. Ageing Res Rev. 2013 Mar;12(2):628-41.

28 Raglio A, Filippi S, Bellandi D, Stramba-Badiale M. Global music approach to persons with dementia: evidence and practice. Clin Interv Aging. 2014 Oct; 9:1669-76. 
29 Kamioka H, Tsutani K, Yamada M, Park H, Okuizumi H, Tsuruoka K, et al. Effectiveness of music therapy: a summary of systematic reviews based on randomized controlled trials of music interventions. Patient Prefer Adherence. 2014 May;8:727-54.

30 Vasionytė I, Madison G. Musical intervention for patients with dementia: a meta-analysis. J Clin Nurs. 2013 May;22(9-10):1203-16.

31 van der Steen JT, van Soest-Poortvliet MC, van der Wouden JC, Bruinsma MS, Scholten RJ, Vink AC. Music-based therapeutic interventions for people with dementia. Cochrane Detabase Syst Rev. 2017 May;5:CD003477.

32 Zhang Y, Cai J, An L, Hui F, Ren T, Ma H, et al. Does music therapy enhance behavioral and cognitive function in elderly dementia patients? A systematic review and meta-analysis. Ageing Res Rev. 2017 May;35:1-11. 